\title{
Partnering with local scientists should be mandatory
}

\author{
Miriam Shuchman, Dawit Wondimagegn, Clare Pain \& Atalay Alem
}

\begin{abstract}
The problem of inequity in international research is perpetuated by policies that enable scientists to conduct research in lowerresourced areas of the world without partnering with local researchers. The World Health Organization (WHO) needs to lead in solving this problem by working with research institutions, journal editors and funding agencies to document the degree of inequity and to impose penalties for failures to collaborate.
\end{abstract}

At the 2013 Global Maternal Health Conference in Arusha, Tanzania, almost exactly a year ago, a panel of health ministers and clinical researchers discussed a provocative plan to ensure fair treatment of biomedical scientists from the poorest countries of the world. The meeting recognized that scientists from wealthy countries continue to run experiments and clinical trials in lower-resourced areas of the world without taking steps to team up with local individuals or focus the research on local problems. In Arusha, a 'code of conduct' was floated that would, among other things, require all scientists who do research in low- and middle-income countries (LMICs) to collaborate with local researchers and include them as coauthors on their papers (Lancet 381, 278, 2013).

There is already a precedent for such measures. Canada, for example, has rules that govern research on humans in its indigenous communities, requiring such projects to engage the relevant community and directing researchers to enhance the research skills of community personnel.

As previously suggested by Jim Campbell, director of ICS Integrare, a research institute based in Barcelona, Spain, the WHO is the natural leader for efforts to encourage the inclusion of local researchers on a global scale. It has a strategy on 'research for health', and one of the strategy's key goals is to set standards for good practice in research. But the WHO needs to do more than further a code of conduct for scientists. It needs to "Equitable treatment of researchers in low- and middle-income countries requires more than just a code of conduct for researchers." students and faculty to conduct career-furthering research in LMICs even when that research does not include local collaborators or coauthors. Most scientific organizations and journals are equally permissive, selecting papers for presentation and publication without regard for whether local collaborators were involved.

Here we offer a few concrete ideas that the WHO can help implement toward the goal of ensuring local experts are included rather than exploited. Firstly, the WHO can work through organizations such as the World Association of Medical Editors and the International Committee of Medical Journal Editors to urge that journals require local authorship for work done in LMICs. In orthopedics, for example, researchers surveying the four top journals found that only $7 \%$ of articles included authors from the developing world (Clin. Orthop. Relat. Res. 470, 2313-2318, 2012), while in psychiatry, a survey of the six top journals found that less than $4 \%$ of articles were from authors in LMICs (Br. J. Psychiatry 190, 77-78, 2007). Already, scientists in low-income countries can submit papers to journals that offer mentoring to LIC authors, such as the Journal of Infection in Developing Countries. At least one journal, the World Journal of Surgery, requires all manuscripts with primary data from LMICs to include a coauthor who lives and works locally.

The WHO could also encourage regulatory bodies overseeing research ethics review to consider local collaboration as a crucial component of ethical research; likewise, it could advocate for research sponsors in work at an institutional level to oblige entities such as universities, journals and funding agencies to document the inequity and quash it.

The challenge of making research more inclusive is real. Some scientists arrive in low-income countries and carry out work without contacting local scientists and institutions-or they include local scientists but marginalize their roles. Local researchers who are sidelined in these scenarios still provide the formal attachment to a local institution and the physical presence necessary for clinical trials by recruiting patients (who trust the locals) and translating study materials, but they are not taken seriously as intellectual contributors. Moreover, they do not own or direct the research, and in some cases their names are not on the subsequent publications.

Scientists from the developed world remain reluctant to help address this inequity. One British researcher who wanted to do research for his master's thesis in Ethiopia approached one of us requesting to gather data through Addis Ababa University but acknowledged that he would not be collaborating with anyone there. He was turned down as a result. But the researcher and his supervisors did not change their plans; another academic unit within the same university accepted his request without a stipulation that he find a local partner.

Meanwhile, research hospitals and universities in developed countries perpetuate this unfortunate situation by permitting their academia and industry to mandate local collaboration as a condition of funding. Some donors, such as Grand Challenges Canada, already require scholars from LMICs as principal investigators. The WHO can help set standards regarding data that journals-and other bodies-should collect to track the problem. For example, clinical trial registries such as ClinicalTrials.gov and the EU Clinical Trials Register may be able to gather information on research relationships by adding data fields to denote the involvement of local scientists.

In short, equitable treatment of researchers in low- and middleincome countries requires more than just a code of conduct for researchers. Scientists must learn that they cannot apply one set of moral standards in LMICs and a different set of standards at home, just as those scientists who are based in developing countries should know that they need not go abroad to be treated as equals.

Miriam Shuchman and Clare Pain are at the Department of Psychiatry, University of Toronto, Toronto, Ontario, Canada. Miriam Shuchman is also at the Clinical and Translational Research Center, University at Buffalo, State University of New York, Buffalo, New York, USA. Dawit Wondimagegn and Atalay Alem are in the College of Health Sciences and the Department of Psychiatry, Addis Ababa University, Addis Ababa, Ethiopia. Clare Pain is also at the Department of Psychiatry, Mount Sinai Hospital, Toronto, Ontario, Canada. 